\title{
Hautklinik Ludwigshafen mit neuer dermatoonkologischer und immunologischer Ausrichtung
}

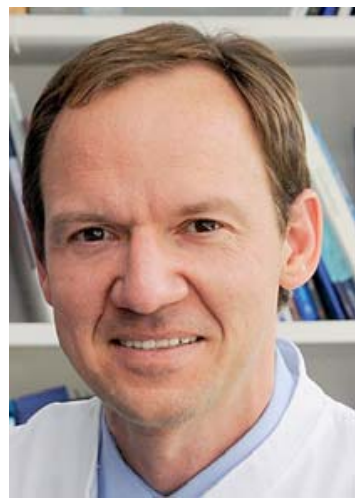

Prof. Dr. Edgar Dippel

\section{Bibliografie}

Dol http://dx.doi.org/ 10.1055/s-0029-1243997

Akt Dermatol 2010; 36; 157 (c) Georg Thieme Verlag KG . Stuttgart $\cdot$ New York ISSN 0340-2541

Korrespondenzadresse

Prof. Dr. Edgar Dippel

Klinikum der Stadt Ludwigshafen $\mathrm{GmbH}$ Bremserstraße 79 67063 Ludwigshafen dippele@klilu.de
Die Hautklinik Ludwigshafen blickt auf eine fast 100-jährige Geschichte zurück, deren Jubiläum wir 2011 feiern werden. Die Ausrichtung der Hautklinik war in der Gründungszeit geprägt von der besonderen Beziehung zur chemischen Industrie bedingt durch die Expansion der Badischen Anilin- und Sodafabrik (BASF). In den Kriegsjahren standen die infektiösen Erkrankungen im Vordergrund. Bereits zu diesem Zeitpunkt wurde eine große Anzahl operativer Eingriffe dokumentiert. Neben einem breiten Angebot der konservativen Dermatologie entwickelte sich bis zum heutigen Zeitpunkt gerade die Dermatochirurgie erheblich weiter. Unter Herrn Prof. Dr. G. Brehm und besonders unter seinem Nachfolger, Herrn Prof. Dr. V. Voigtländer, der die Klinik bis Januar 2008 leitete, wurde der dermatochirurgische Schwerpunkt weiter ausgebaut. In der Gegenwart wird das Fachgebiet Dermatologie und Venerologie von zwei wichtigen klinisch-wissenschaftlichen Ausrichtungen geprägt, die von den Bedürfnissen der steigenden Patientenzahl, von Innovationen, wissenschaftlichen Entwicklungen und Qualitätsgedanken bedingt werden. Dabei handelt es sich um dermatoonkologische und immunologische Aspekte, die das Gesicht der Dermatologie insgesamt in den letzten Jahren verändert haben. Die stetig zunehmende Inzidenz der Hauttumoren, aber auch das Bestreben, verbindliche Standards bei der Behandlung bösartiger Hauterkrankungen festzuschreiben, hat zur Etablierung von Hauttumorzentren geführt. Diese sollen die Voraussetzungen schaffen, Therapiestrategien zu optimieren und die Überlebensraten bei Hauttumorpatienten zu verbessern. Die von der Deutschen Krebsgesellschaft herausgegebenen fachlichen Anforderungen und Bedingungen an ein QualitätsmanagementSystem sind der Grund dafür, dass sich Hautkliniken, die diese Zertifizierung anstreben, neu strukturieren müssen. Auch die Hautklinik Ludwigshafen hat diesen Zertifizierungsprozess durchlaufen und ist seit Juli 2009 Hauttumorzentrum Rheinpfalz. Logistische und organisatorische Prozessabläufe wurden optimiert, ein Tumordokumentationssystem eingeführt, eine psychoonkologische Mitbetreuung etabliert und „study-nurses“ für die Begleitung von Studienpatienten in ein umfassendes dermatoonkologisches Gesamtkonzept einbezogen. Das Angebot an aktuellen Studien der ADO, der EORTC-Melanoma Group und der EORTC Cutaneous Lymphoma Task Force (CLTF) ist zum wichtigen Bestandteil des Hauttumorzentrums Ludwigshafen geworden. Kooperationen mit den Hautkliniken Mainz, Mannheim und Heidelberg ergänzen hier das Therapiespektrum.

In enger Zusammenarbeit mit den niedergelassenen Kollegen und den interdisziplinären Koopera- tionspartnern wurde ein Netzwerk etabliert, das von den Patienten mit Wertschätzung angenommen worden ist. Die Patientenzahlen im stationären Bereich haben sich im Jahr 2008 um 20\% auf 2132 gesteigert.

Ein weiterer Schwerpunkt ist die Immunologie und das damit verbundene Angebot an Immuntherapien bei entzündlichen Erkrankungen. In enger Zusammenarbeit mit der rheumatologischen Abteilung der Medizinischen Klinik werden Patienten mit Sklerodermie, Dermatomyositis, Lupus erythematodes, Vaskulitiden und Psoriasisarthritis gemeinsam betreut und auch hier wird die gesamte Breite der immunologischen Therapiemöglichkeiten und Studien angeboten.

Das gesamte Ärzteteam ist hochqualifiziert und motiviert. Die Weiterbildungsermächtigung liegt für Dermatologie und Venerologie, Allergologie, Phlebologie und medikamentöse Tumortherapie vor. Leitender Oberarzt ist Dr. C. Löser, der mehrere Zusatzqualifikationen einbringt und z.B. Operationen am Nagelorgan auf höchstem Niveau durchführt. Neben seinem Engagement für die operative Dermatologie im Vorstand der Deutschen Gesellschaft für Dermatochirurgie gilt sein Interesse auch der Geschichte der Dermatologie. In Zusammenarbeit mit Herrn Prof. Dr. med. Dr. h.c. mult. Gerd Plewig wurde 2008 das umfassende Werk „Pantheon der Dermatologie“ herausgegeben. Spezialgebiete von Frau Dr. K. Niedergethmann, Oberärztin der Hautklinik, sind die Bereiche Phlebologie, Sonografie, Berufsdermatologie und die Therapie entzündlicher Erkrankungen; Oberärztin Frau Dr. C. Coordes ist die Zentrumskoordinatorin des Hauttumorzentrums und weiterbildungsberechtigt für den Bereich der Allergologie. Zum jungen und engagierten Team der Rotationsassistenten und Stationsärzte zählen derzeit Frau Dr. A. Ha (Fachärztin), Frau Dr. F. Brinkmann, Frau Dr. E. Kühl, Frau M. Groß, Frau Dr. S. Navysany, Frau M. Konrad und Frau N. Müller. Fortund Weiterbildung sind ein zentrales Anliegen. Dies äußert sich auch in der erfolgreichen Einführung von Rotationen der Assistenten auf den stationären Sektoren und in den Spezialsprechstunden.

Die Hautklinik profitiert vom richtungsweisenden „Ludwigshafener Modell“, bei dem die Kodierung nach DRG-Gesichtspunkten zentral von einer Stabsstelle der Geschäftsführung übernommen wurde. Die Ludwigshafener Hautklinik wächst personell aufgrund der Leistungssteigerungen und wird schrittweise, auch räumlich, weiter modernisiert, um den neuen Anforderungen und Herausforderungen unserer Patienten und der Gesundheitslandschaft gerecht zu werden.

Mit den besten kollegialen Grüßen 